\title{
Common fixed points in partially ordered modular function spaces
}

\author{
Mujahid Abbas', Sartaj Ali and Poom Kumam³
}

\author{
"Correspondence: \\ poom.kum@kmutt.ac.th \\ ${ }^{3}$ Department of Mathematics, \\ Faculty of Science, King Mongkut's \\ University of Technology Thonburi \\ (KMUTT), Bang Mod, Thrung Khru, \\ Bangkok, 10140, Thailand \\ Full list of author information is \\ available at the end of the article
}

\begin{abstract}
The purpose of this paper is to study the existence and uniqueness of common fixed point results in partially ordered modular function spaces.
\end{abstract}

MSC: $47 \mathrm{H} 10 ; 54 \mathrm{H} 25 ; 54 \mathrm{C} 60 ; 46 \mathrm{~B} 40$

Keywords: fixed point; ordered modular function space

\section{Introduction}

Study of modular spaces was initiated by Nakano [1] in connection with the theory of order spaces which was further generalized by Musielak and Orlicz [2]. The study of fixed points of mappings on complete metric spaces equipped with a partial ordering $\preceq$ was first investigated in 2004 by Ran and Reurings [3], and then by Nieto and Rodriguez-Lopez [4]. They applied their results to obtain a unique solution for a first order ordinary differential equation with periodic boundary conditions (see also [5]). The study of this theory in the context of modular function spaces was initiated by Khamsi et al. [6] (see also [7] and [8]). Kuaket and Kumam [9] and Mongkolkeha and Kumam [10-12], considered and proved some fixed point and common fixed point results for generalized contraction mappings in modular spaces. Also, Kumam [13] obtained some fixed point theorems for non-expansive mappings in arbitrary modular spaces. Recently, Kutabi and Latif [14] studied fixed points of multivalued maps in modular function spaces.

The study of common fixed points of mappings satisfying certain contractive conditions in the setup of partially ordered metric spaces can be employed to establish the existence of solutions of many types of operator equations, such as differential and integral equations. There are a few examples given in the following papers: [15-20] and references mentioned therein. The objective of this paper is to initiate the study of common fixed point results in partially ordered modular function spaces. As an application of our results, we study the property $Q$ for mappings involved herein.

\section{Preliminaries}

Some basic facts and notations about modular spaces are recalled from [21].

Definition 2.1 Let $X$ be a real (or complex) vector space. A functional $\rho: X \rightarrow[0, \infty]$ is called modular if, for any $x, y$ in $X$, the following hold:

$\left(\mathrm{m}_{1}\right) \rho(x)=0$ if and only if $x=0$.

$\left(\mathrm{m}_{2}\right) \rho(\alpha x)=\rho(x)$ for every scalar $\alpha$ with $|\alpha|=1$.

$\left(\mathrm{m}_{3}\right) \rho(\alpha x+\beta y) \leq \rho(x)+\rho(y)$ provided that $\alpha+\beta=1$, and $\alpha, \beta \geq 0$. 
If $\left(\mathrm{m}_{3}\right)$ is replaced by $\rho(\alpha x+\beta y) \leq \alpha \rho(x)+\beta \rho(y)$ if $\alpha+\beta=1$, and $\alpha, \beta \geq 0$, then $\rho$ is called convex modular.

The vector space $X_{\rho}$ given by

$$
X_{\rho}=\{x \in X ; \rho(\lambda x) \rightarrow 0 \text { as } \lambda \rightarrow 0\}
$$

is called a modular space. Generally, the modular $\rho$ is not subadditive and therefore does not behave as a norm or a distance.

Modular space $X_{\rho}$ can be equipped with an $F$-norm defined by

$$
\|x\|_{\rho}=\inf \left\{\alpha>0 ; \rho\left(\frac{x}{\alpha}\right) \leq \alpha\right\} .
$$

If $\rho$ is convex modular, then

$$
\|x\|_{\rho}=\inf \left\{\alpha>0 ; \rho\left(\frac{x}{\alpha}\right) \leq 1\right\}
$$

defines a norm on the modular space $X_{\rho}$ and is called the Luxemburg norm.

Define the $\rho$-ball, centered at $x \in X_{\rho}$ with radius $r$, as

$$
B_{\rho}(x, r)=\{h \in X \rho ; \rho(x-h) \leq r\} .
$$

Definition 2.2 A function modular is said to satisfy $\Delta_{2}$-type condition, if there exists $K>0$ such that for any $x \in X_{\rho}$, we have $\rho(2 x) \leq K \rho(x)$.

Definition 2.3 $\rho$ is said to satisfy the $\Delta_{2}$-condition if $\rho\left(2 x_{n}\right) \rightarrow 0$ whenever $\rho\left(x_{n}\right) \rightarrow 0$ as $n \rightarrow \infty$.

Definition 2.4 Let $X_{\rho}$ be a modular space. The sequence $\left\{x_{n}\right\} \subset X_{\rho}$ is called:

$\left(\mathrm{t}_{1}\right) \rho$-convergent to $x \in X_{\rho}$, if $\rho\left(x_{n}-x\right) \rightarrow 0$ as $n \rightarrow \infty$.

(t $\left.\mathrm{t}_{2}\right) \rho$-Cauchy, if $\rho\left(x_{n}-x_{m}\right) \rightarrow 0$ as $n$ and $m \rightarrow \infty$.

Note that $\rho$-convergence does not imply $\rho$-Cauchy since $\rho$ does not satisfy the triangle inequality. In fact, one can show that this will happen if and only if $\rho$ satisfies the $\Delta_{2}$-type condition.

It is well known that $[6,22]$ under the $\Delta_{2}$-condition the norm convergence and modular convergence are equivalent. The same is true when we deal with the $\Delta_{2}$-type condition. Throughout this paper, we assume that modular function $\rho$ is convex and satisfies the $\Delta_{2}$-type condition. We also state the following definition and results given in [7].

Definition 2.5 The growth function $w_{\rho}$ of a function modular $\rho$ is defined as

$$
w_{\rho}(t)=\sup \left\{\frac{\rho(t x)}{\rho(x)}, x \in X_{\rho} \backslash\{0\}\right\} \text { for all } 0 \leq t<\infty
$$

Observe that $w_{\rho}(t) \leq 1$ for all $t \in[0,1]$. 
Lemma 2.6 The growth function $\omega$ has the following properties:

$\left(\mathrm{g}_{1}\right) \omega(t)<\infty$, for each $t \in[0, \infty)$.

$\left(\mathrm{g}_{2}\right) \omega:[0, \infty) \rightarrow[0, \infty)$ is a convex, strictly increasing function. So, it is continuous.

(g. $\omega(\alpha \beta) \leq \omega(\alpha) \omega(\beta)$; for all $\alpha, \beta \in[0, \infty)$.

( $\left.\mathrm{g}_{4}\right) \omega^{-1}(\alpha) \omega^{-1}(\beta) \leq \omega^{-1}(\alpha \beta)$; for all $\alpha \beta \in[0, \infty)$, where $\omega^{-1}$ is the inverse function of $\omega$.

The following lemma shows that the growth function can be used to give an upper bound for $\|x\|_{\rho}$ for each $x \in X_{\rho}$.

Lemma 2.7 Let $\rho$ be a convex modular function satisfying the $\Delta_{2}$-type condition. Then

$$
\|x\|_{\rho} \leq \frac{1}{\omega^{-1}\left(\frac{1}{\rho(x)}\right)}
$$

whenever $x \in X_{\rho}$.

Let $S$ and $T$ be two self-maps on a modular function space $X_{\rho}$. A point $x \in X_{\rho}$ is called (1) a fixed point of $S$ if $S(x)=x$; (2) a coincidence point of a pair (S,T) if $S x=T x$; (3) a common fixed point of a pair $(S, T)$ if $x=S x=T x$. If $w=S x=T x$ for some $x$ in $X_{\rho}$, then $w$ is called a point of coincidence of $S$ and $T$.

The pair $(S, T)$ is said to be compatible if $\rho\left(S T x_{n}-T S x_{n}\right) \rightarrow 0$ as $n \rightarrow \infty$, whenever $\left\{x_{n}\right\}$ is a sequence in $X$ such that $\left\{S x_{n}\right\}$ and $\left\{T x_{n}\right\}$ are $\rho$-convergent to $t \in X_{\rho}$.

A pair $(S, T)$ is said to be $\rho$-weakly compatible if $S$ and $T$ commute at their coincidence points.

We denote the set of fixed points of $S$ by Fix $(S)$.

Definition 2.8 Let $\left(X_{\rho}, \preccurlyeq\right)$ be a partially modular ordered space. A pair $\left(T_{1}, T_{2}\right)$ of selfmaps of $X_{\rho}$ is said to be $\rho$-weakly increasing if $T_{1}(f) \leq T_{2} T_{1}(f)$ and $T_{2}(f) \leq T_{1} T_{2}(f)$ for all $f \in X_{\rho}$.

Definition 2.9 Let $\left(X_{\rho}, \preccurlyeq\right)$ be a partially modular ordered space and $T_{1}, T_{2}$ be two selfmaps on $X_{\rho}$. An order pair $\left(T_{1}, T_{2}\right)$ is said to be partially $\rho$-weakly increasing if $T_{1}(f) \leq$ $T_{2} T_{1}(f)$ for all $f \in X_{\rho}$.

The pair $\left(T_{1}, T_{2}\right)$ is $\rho$-weakly increasing if and only if the ordered pairs $\left(T_{1}, T_{2}\right)$ and $\left(T_{2}, T_{1}\right)$ are partially $\rho$-weakly increasing.

Definition 2.10 Let $\left(X_{\rho}, \preccurlyeq\right)$ be a partially modular ordered space. A mapping $T_{1}$ is said to be $\rho$-weak annihilator of $T_{2}$ if $T_{1} T_{2}(f) \leq f$ for all $f \in x_{\rho}$.

Definition 2.11 Let $\left(X_{\rho}, \preccurlyeq\right)$ be a partially ordered modular space. A mapping $T_{1}$ is said to be $\rho$-dominating if $f \leq T_{1} f$ for all $f \in X_{\rho}$.

Definition 2.12 Let $\left(X_{\rho}, \preccurlyeq\right)$ be a partially modular ordered space and $T_{1}, T_{2}, T_{3}$ be three self-maps on $X_{\rho}$, such that $T_{1} X_{\rho} \subseteq T_{3} X_{\rho}$ and $T_{2} X_{\rho} \subseteq T_{3} X_{\rho}$. We say that $T_{1}$ and $T_{2}$ are $\rho$-weakly increasing with respect to $T_{3}$ if and only if for all $f \in X_{\rho}$, we have $T_{1} f \leq T_{2} g$ for all $g \in T_{3}^{-1}\left(T_{1} f\right)$, and $T_{2} f \leq T_{1} g$, for all $g \in T_{3}^{-1}\left(T_{2} f\right)$, where $T_{3}^{-1}(f)=\left\{h \in X_{\rho} \mid T_{3} h=f\right\}$ for all $f \in X_{\rho}$. 
Definition 2.13 Let $\left(X_{\rho}, \preccurlyeq\right)$ be a partially modular ordered space and $T_{1}, T_{2}, T_{3}$ be three self-maps on $X_{\rho}$ such that $T_{1} X_{\rho} \subseteq T_{3} X_{\rho}$. We say that $T_{1}$ and $T_{2}$ are partially $\rho$-weakly increasing with respect to $T_{3}$ if for all $f \in X_{\rho}$, we have $T_{1} f \leq T_{2} g$, for all $g \in T_{3}^{-1}\left(T_{1} f\right)$.

Definition 2.14 Let $X$ be a vector space. Then $(X, \preceq, \rho)$ is called an ordered modular function space iff: (i) $\rho$ is convex modular function on $X$ and (ii) $\preceq$ is a partial order on $X$.

Let $(X, \preceq)$ be a partial ordered set. Then $x, y \in X$ are called comparable if $x \preceq y$ or $y \preceq x$ holds.

\section{Common fixed point results}

We begin with a common fixed point theorem for two pairs of partially weakly increasing functions on an ordered modular function spaces. It may regarded as the main result of this article.

Theorem 3.1 Let $(X, \preceq, \rho)$ be a complete ordered modular function space and $S, I, T$, and $J$ self-maps on $X_{\rho}$ such that $S\left(X_{\rho}\right) \subseteq J\left(X_{\rho}\right)$ and $I\left(X_{\rho}\right) \subseteq T\left(X_{\rho}\right)$. Suppose that $(J, S)$ and $(I, T)$ are $\rho$-weakly increasing, and the dominating maps $S$ and $T$ are weak annihilators of $J$ and $I$, respectively. If for every two comparable elements $f, g \in X_{\rho}$

$$
\rho(S f-T g) \leq \alpha \rho(I f-J g)
$$

is satisfied, then $S, I, T$, and J have a common fixed point provided that for a non-decreasing sequence $\left\{f_{n}\right\}$ with $f_{n} \leq g_{n}$ for all $n$ and $g_{n} \rightarrow g$ implies that $f_{n} \leq g$ and either

(a) $\{S, I\}$ are $\rho$-compatible, $S$ or I is $\rho$-continuous and $\{T, J\}$ are $\rho$-weakly compatible;

(b) $\{T, J\}$ are $\rho$-compatible, $T$ or $J$ is $\rho$-continuous and $\{S, I\}$ are $\rho$-weakly compatible.

Moreover, the set of common fixed points of $S, I, T$, and $J$ is well ordered if and only if $S, I$, $T$, and $J$ have one and only one common fixed point.

Proof (a) Let $f_{0} \in X_{\rho}$. Construct sequences $f_{n}$ and $g_{n}$ in $X_{\rho}$, such that $g_{2 n-1}=S f_{2 n-2}=J f_{2 n-1}$ and $g_{2 n}=T f_{2 n-1}=I f_{2 n}$. This can be done because $S\left(X_{\rho}\right) \subseteq J\left(X_{\rho}\right)$ and $I\left(X_{\rho}\right) \subseteq T\left(X_{\rho}\right)$. Since $S$ is a $\rho$-dominating map and the pair $(J, S)$ is partially $\rho$-weakly increasing so $f_{2 n-2} \leq S f_{2 n-2}=$ $J f_{2 n-1} \leq S\left(J f_{2 n-1}\right)$. Also, $S$ is a $\rho$-weak annihilator of $J$ so $f_{2 n-2} \leq S f_{2 n-2}=J f_{2 n-1} \leq S\left(J f_{2 n-1}\right) \leq$ $f_{2 n-1}$. This implies that $f_{2 n-2} \leq f_{2 n-1}$. Since $T$ is a dominating map, $f_{2 n-1} \leq T f_{2 n-1}=I f_{2 n}$. As $(I, T)$ is a pair of partially weakly increasing mappings, $I f_{2 n} \leq T\left(I f_{2 n}\right)$ and $f_{2 n-1} \leq T f_{2 n-1}=$ $I f_{2 n} \leq T\left(I f_{2 n}\right)$. Also $T$ is a weak annihilator of $I$, so we have $f_{2 n-1} \leq T f_{2 n-1}=I f_{2 n} \leq T\left(I f_{2 n}\right) \leq$ $f_{2 n}$. This implies that $f_{2 n-1} \leq f_{2 n}$. Hence for all $n \geq 1$ we have $f_{n} \leq f_{n+1}$. Suppose that $\rho\left(g_{2 n}-\right.$ $\left.g_{2 n+1}\right)>0$ for every $n$. If not, then $\rho\left(g_{2 n}-g_{2 n+1}\right)=0$ implies that $g_{2 n}-g_{2 n+1}=0$, that is, $g_{2 n}=g_{2 n+1}$ for some $n$. Now from inequality (3.1) we have

$$
\rho\left(g_{2 n+1}-g_{2 n+2}\right)=\rho\left(S f_{2 n}-T f_{2 n+1}\right) \leq \alpha \rho\left(I f_{2 n}-I f_{2 n+1}\right)=\alpha \rho\left(g_{2 n}-g_{2 n+1}\right)
$$

and therefore $\rho\left(g_{2 n+1}-g_{2 n+2}\right)=0$. So $g_{2 n+1}=g_{2 n+2}$ and so on. Thus $\left\{g_{n}\right\}$ becomes a constant sequence and $g_{2 n}$ is a required common fixed point of given mappings. Assume that $\rho\left(g_{2 n+1}-g_{2 n+2}\right) \neq 0$ for each $n$. From (3.1), we obtain

$$
\begin{aligned}
\rho\left(g_{2 n+1}-g_{2 n+2}\right) & =\rho\left(S f_{2 n}-T f_{2 n+1}\right) \\
& \leq \alpha \rho\left(I f_{2 n}-J f_{2 n+1}\right)=\alpha \rho\left(g_{2 n}-g_{2 n+1}\right)
\end{aligned}
$$




$$
\begin{aligned}
& =\alpha \rho\left(S f_{2 n-1}-T f_{2 n}\right) \\
& \leq \alpha^{2} \rho\left(I f_{2 n-1}-J f_{2 n}\right)=\alpha^{2} \rho\left(g_{2 n-1}-g_{2 n}\right) \\
& =\alpha^{2} \rho\left(S f_{2 n-2}-T f_{2 n-1}\right) \leq \alpha^{3} \rho\left(I f_{2 n-2}-J f_{2 n-1}\right) \\
& =\alpha^{3}\left(g_{2 n-2}-g_{2 n-1}\right)=\alpha^{3} \rho\left(S f_{2 n-3}-T f_{2 n-2}\right) \\
& \leq \alpha^{4} \rho\left(I f_{2 n-3}-J f_{2 n-2}\right)=\alpha^{4} \rho\left(g_{2 n-3}-g_{2 n-2}\right) .
\end{aligned}
$$

Inductively, we have $\rho\left(g_{2 n+1}-g_{2 n+2}\right) \leq \alpha^{n} \rho\left(g_{n+1}-g_{n+2}\right)$, which implies that

$$
\frac{1}{\alpha^{n} \rho\left(g_{n+1}-g_{n+2}\right)} \leq \frac{1}{\rho\left(g_{2 n+1}-g_{2 n+2}\right)} .
$$

Using Lemma 2.7, we have

$$
\left\|g_{2 n+1}-g_{2 n+2}\right\|_{\rho} \leq \frac{1}{\omega^{-1}\left(\frac{1}{g_{2 n+1}-g_{2 n+2}}\right)}
$$

and

$$
\omega^{-1}\left(\frac{1}{\alpha^{n} \rho\left(g_{n+1}-g_{n+2}\right)}\right) \leq \omega^{-1}\left(\frac{1}{\rho\left(g_{2 n+1}-g_{2 n+2}\right)}\right) .
$$

Employing the properties of the growth function, we obtain

$$
\omega^{-1}\left(\frac{1}{\alpha}\right)^{n} \omega^{-1}\left(\frac{1}{\rho\left(g_{n+1}-g_{n+2}\right)}\right) \leq \omega^{-1}\left(\frac{1}{\rho\left(g_{2 n+1}-g_{2 n+2}\right)}\right),
$$

which implies that

$$
\begin{aligned}
\left\|g_{2 n+1}-g_{2 n+2}\right\|_{\rho} & \leq \frac{1}{\omega^{-1}\left(\frac{1}{\alpha}\right)^{n} \omega^{-1}\left(\frac{1}{\rho\left(g_{n+1}-g_{n+2}\right)}\right)} \\
& =\frac{1}{\left[\omega^{-1}\left(\frac{1}{\alpha}\right)\right]^{n} \omega^{-1}\left(\frac{1}{\rho\left(g_{n+1}-g_{n+2}\right)}\right)} .
\end{aligned}
$$

As $\omega(1)=1$, and $\alpha \lessdot 1$ so $1 \lessdot \omega^{-1}\left(\frac{1}{\alpha}\right)$, and $\frac{1}{\omega^{-1}\left(\frac{1}{\alpha}\right)}<1$. This shows that $\left\{g_{2 n}\right\}$ is a Cauchy sequence in $\left(X_{\rho},\|\cdot\|_{\rho}\right)$. There exists $h \in X_{\rho}$ such that $\left\|g_{2 n}-h\right\|_{\rho} \rightarrow 0$. That is, the sequence $\left\{g_{2 n}\right\}$ is norm convergent to $h \in X_{\rho}$. Since the $\Delta_{2}$-condition implies equivalence of norm and modular convergence, $\left\{g_{2 n}\right\}$ is modular convergent to $h \in X_{\rho}$. Therefore $\left\{g_{2 n}\right\} \stackrel{\rho}{\rightarrow} h$. Thus, we have $h=\lim _{n \rightarrow \infty} g_{2 n+1}=\lim _{n \rightarrow \infty} J f_{2 n+1}=\lim _{n \rightarrow \infty} S f_{2 n}$ and $h=$ $\lim _{n \rightarrow \infty} g_{2 n+2}=\lim _{n \rightarrow \infty} T f_{2 n+1}=\lim _{n \rightarrow \infty} I f_{2 n+2}$. Assume that $I$ is continuous. Since $\{S, I\}$ are $\rho$-compatible, we have $\lim _{n \rightarrow \infty} S I f_{2 n+2}=\lim _{n \rightarrow \infty} I S f_{2 n+2}=I h$. As $T$ is a $\rho$-dominating map, $f_{2 n+1} \leq T f_{2 n+1}=I f_{2 n+2}$, that is, $f_{2 n+1} \leq I f_{2 n+2}$. Therefore we have

$$
\rho\left(\operatorname{SIf}_{2 n+2}-I f_{2 n+2}\right)=\rho\left(\operatorname{SIf}_{2 n+2}-T f_{2 n+1}\right) \leq \alpha \rho\left(I I f_{2 n+2}-J f_{2 n+1}\right),
$$

which on taking the limit as $h \rightarrow \infty$ gives $\rho(I h-h) \leq \alpha \rho(I h-h)$. That is, $(1-\alpha) \rho(I h-h) \leq 0$. As $\alpha<1$, so $\rho(I h-h) \leq 0$ implies that $I h=h$. Since $T$ is $\rho$-dominating, $f_{2 n+1} \leq T f_{2 n+1}$. Also, 
$T f_{2 n+1}=g_{2 n+2} \rightarrow h$ as $n \rightarrow \infty$ implies that $f_{2 n+1} \leq h$. So, we have

$$
\begin{aligned}
\rho\left(S h-g_{2 n+2}\right) & =\rho\left(S h-I f_{2 n+2}\right)=\rho\left(S h-T f_{2 n+1}\right) \\
& \leq \alpha \rho\left(I h-I f_{2 n+1}\right)=\alpha \rho\left(I h-g_{2 n+1}\right) .
\end{aligned}
$$

On taking the limit as $n \rightarrow \infty$, we obtain

$$
\rho(S h-h) \leq \alpha \rho(I h-h)=0 .
$$

Hence $\rho(S h-h)=0$ and $S h=h$. As $S\left(X_{\rho}\right) \subseteq J\left(X_{\rho}\right)$, there exists a point $k \in X_{\rho}$ such that $S h=$ $J k$. Suppose that $T k \neq J k$. Since $S$ is $\rho$-dominating, $(J, S)$ is partially $\rho$-weakly increasing, and $S$ is a $\rho$-weak annihilator of $J$, so we have $h \leq S h=J k \leq S J k \leq k$, that is, $h \leq k$. Thus, we have

$$
\begin{aligned}
\rho(h-T k) & =\rho(J k-T k)=\rho(S h-T k) \\
& \leq \alpha \rho(I h-J k)=\alpha \rho(h-J k)=0
\end{aligned}
$$

giving $h=T k$. Since $\{T, J\}$ are $\rho$-weakly compatible, $T h=T S h=T J k=J T k=J h$. Thus $h$ is a coincidence point of $T$ and $J$. As $S$ is a $\rho$-dominating map, $f_{2 n} \leq S f_{2 n}$. Now $S f_{2 n} \rightarrow h$ as $n \rightarrow \infty$ implies that $f_{2 n} \leq h$. Now from (3.1), we have

$$
\rho\left(S f_{2 n}-T h\right) \leq \alpha \rho\left(I f_{2 n}-J h\right)
$$

which, on taking the limit as $n \rightarrow \infty$, gives

$$
\begin{aligned}
& \rho(h-T h) \leq \alpha \rho(h-J h)=\alpha \rho(h-T h), \\
& \rho(h-T h) \leq \alpha \rho(h-T h)
\end{aligned}
$$

or $(1-\alpha) \rho(h-T h) \leq 0$, as $\alpha<1$, so $h=T h$. Thus, $S h=I h=T h=J h=h$. That is, $h$ is a common fixed point of $S, T, I$, and $J$.

(b) Similarly the result follows when (b) holds.

Now suppose that the set of common fixed points of $S, I, T$, and $J$ is well ordered. We claim that the common fixed point of $S, I, T$, and $J$ is unique. Assume to the contrary that these maps have two common fixed points $u$ and $v$, that is,

$$
S u=I u=T u=J u=u \quad \text { and } \quad S v=I v=T v=J v=v .
$$

From inequality (3.1), we have

$$
\rho(u-v)=\rho(S u-T v) \leq \alpha \rho(I u-J v)=\alpha \rho(u-v) .
$$

Thus

$$
(1-\alpha) \rho(u-v) \leq 0 \text { implies that } \rho(u-v)=0 \text {, which further implies that } u=v \text {. }
$$

Hence uniqueness is proved. The converse is straightforward. 
Corollary 3.2 Let $(X, \preceq, \rho)$ be a complete ordered modular function space and $S, I$, and $J$ self-maps on $X$ such that $S(X) \subseteq J(X)$, and $I(X) \subseteq S(X)$. Suppose that $(J, S)$ and $(I, S)$ are partially $\rho$-weakly increasing and the dominating map $S$ is a weak annihilator of $J$ and $I$. If for every two comparable elements $f, g \in X$

$$
\rho(S f-S g) \leq \alpha \rho(I f-J g)
$$

is satisfied, then $S, I$, and J have a common fixed point provided that for a non-decreasing sequence $\left\{f_{n}\right\}$ with $f_{n} \leq g_{n}$ for all $n$ and $g_{n} \rightarrow g$ implies that $f_{n} \leq g$ and either

(a) $\{S, I\}$ are $\rho$-compatible, $S$ or I is $\rho$-continuous and $\{S, J\}$ are $\rho$-weakly compatible;

(b) $\{S, J\}$ are $\rho$-compatible, $S$ or $J$ is $\rho$-continuous and $\{S, I\}$ are $\rho$-weakly compatible.

Moreover, the set of the common fixed points of $S, I$, and $J$ is well ordered if and only if $S, I$, and $J$ have one and only one common fixed point.

Corollary 3.3 Let $(X, \preceq, \rho)$ be a complete ordered modular function space and $S, T$, and $J$ self-maps on $X$ such that $S\left(L_{\rho}\right) \subseteq J\left(L_{\rho}\right)$ and $J\left(L_{\rho}\right) \subseteq T\left(L_{\rho}\right)$. Suppose that $(J, S)$ and $(J, T)$ are partially $\rho$-weakly increasing, and the dominating maps $S$ and $T$ are weak annihilators of $J$. If for every two comparable elements $f, g \in L_{\rho}$

$$
\rho(S f-T g) \leq \alpha \rho(J f-J g)
$$

is satisfied, then $S, T$, and J have a common fixed point provided that for a non-decreasing sequence $\left\{f_{n}\right\}$ with $f_{n} \leq g_{n}$ for all $n$ and $g_{n} \rightarrow g$ implies that $f_{n} \leq g$ and either

(a) $\{S, J\}$ are $\rho$-compatible, $S$ or $J$ is $\rho$-continuous and $\{T, J\}$ are $\rho$-weakly compatible;

(b) $\{T, J\}$ are $\rho$-compatible, $T$ or $J$ is $\rho$-continuous and $\{S, J\}$ are $\rho$-weakly compatible.

Moreover, the set of common fixed points of $S, T$, and $J$ is well ordered if and only if $S, T$, and $J$ have one and only one common fixed point.

Corollary 3.4 Let $(X, \preceq, \rho)$ be a complete ordered modular function space, $S$ and $J$ selfmaps on $X$ such that $S\left(L_{\rho}\right) \subseteq J\left(L_{\rho}\right)$ and $J\left(L_{\rho}\right) \subseteq S\left(L_{\rho}\right)$. Suppose that $(J, S)$ is a partially $\rho$-weakly increasing and the dominating map $S$ is a weak annihilator of J. Iffor every two comparable elements $f, g \in L_{\rho}$

$$
\rho(S f-S g) \leq \alpha \rho(J f-J g)
$$

is satisfied, then $S$ and $J$ have a common fixed point provided that for a non-decreasing sequence $\left\{f_{n}\right\}$ with $f_{n} \leq g_{n}$ for all $n$ and $g_{n} \rightarrow g$ it is implied that $f_{n} \leq g$ and either $\{S, J\}$ are $\rho$-compatible, $S$ or $J$ is $\rho$-continuous and $\{S, J\}$ are $\rho$-weakly compatible. Moreover, the set of common fixed points of $S$ and $J$ is well ordered if and only if $S$ and $J$ have one and only one common fixed point.

Theorem 3.5 Let $(X, \preceq, \rho)$ be a complete ordered modular function space and $S, T, I$, and $J$ continuous self-maps on $X_{\rho}$. Suppose that $(S, I)$ and $(T, J)$ are $\rho$-compatible, $(S, T)$ and $(T, S)$ are $\rho$-partially weakly increasing with respect to $J$ and $I$, respectively, and

$$
\rho(S f-T g) \leq \alpha \rho(I f-J g)
$$


holds for every $f, g \in X_{\rho}$ for which If and Ig are comparable. Then the pairs $(S, I)$ and $(T, J)$ have a coincidence point $h \in X$. Moreover if Ih and Jh are comparable, then $h \in X$ is a coincidence point of $S, T, I$, and $J$.

Proof Let $f_{0}$ be an arbitrary point in $X$. Construct the sequences $\left\{f_{n}\right\}$ and $\left\{g_{n}\right\}$ in $X$ such that $g_{2 n}=S f_{2 n}=J f_{2 n+1}$ and $g_{2 n+1}=T f_{2 n+1}=I f_{2 n+2}$. As $(S, T)$ is $\rho$-partially weakly increasing with respect to $J$, from $f_{2 n+1} \in J^{-1}\left(S f_{2 n}\right)$ we have

$$
J f_{2 n+1}=S f_{2 n} \leq T f_{2 n+1}=I f_{2 n+2} .
$$

Since $(T, S)$ is $\rho$-partially weakly increasing with respect to $I$, from $f_{2 n+2} \in I^{-1}\left(T f_{2 n+1}\right)$, we have

$$
I f_{2 n+2}=T f_{2 n+1} \leq S f_{2 n+2}=J f_{2 n+3} .
$$

Hence $J f_{1} \leq I f_{2} \leq I f_{3} \leq \cdots \leq I f_{2 n+1} \leq I f_{2 n+2} \leq J f_{2 n+3} \leq \cdots$, that is, $g_{0} \leq g_{1} \leq g_{2} \leq \cdots \leq g_{2 n} \leq$ $g_{2 n+1} \leq g_{2 n+2} \cdots$. Following similar arguments to those given in Theorem 3.1, we obtain $\lim _{n \rightarrow \infty} \rho\left(g_{n}-g_{n+1}\right)=0$ and $\left\{g_{n}\right\}$ is a Cauchy sequence. Now we show the existence of the coincidence point for the pairs $(S, I)$ and $(T, J)$. To prove this, we proceed as follows: Since $X$ is complete, there exists $h \in X_{\rho}$ such that $\lim _{n \rightarrow \infty} g_{n}=h$. That is, $\lim _{n \rightarrow \infty} \rho\left(I f_{2 n}-h\right)=$ $\lim _{n \rightarrow \infty} \rho\left(S f_{2 n}-h\right)=\lim _{n \rightarrow \infty} \rho\left(I f_{2 n+2}-h\right)=\lim _{n \rightarrow \infty} \rho\left(T f_{2 n+1}-h\right)=\lim _{n \rightarrow \infty} \rho\left(J f_{2 n+1}-h\right)=0$. By compatibility of $(S, I)$ and $(T, J)$, we have

$$
\lim _{n \rightarrow \infty} \rho\left(I\left(S f_{2 n}\right)-S\left(I f_{2 n}\right)\right)=\lim _{n \rightarrow \infty} \rho\left(J\left(T f_{2 n+1}\right)-T\left(J f_{2 n+1}\right)\right)=0 .
$$

By continuity of $S, T, I$, and $J$, we have $\lim _{n \rightarrow \infty} \rho\left(S\left(I f_{2 n}\right)-S h\right)=\lim _{n \rightarrow \infty} \rho\left(T\left(J f_{2 n+1}\right)-\right.$ $T h)=0$. Note that

$$
\begin{aligned}
\rho(I h-S h) & \leq \omega(3) \rho\left(I h-I S f_{2 n}\right)+\omega(3) \rho\left(I S f_{2 n}-S I f_{2 n}\right)+\omega(3) \rho\left(S I f_{2 n}-S h\right) \\
& =\omega(3)\left[\rho\left(I h-I S f_{2 n}\right)+\rho\left(I S f_{2 n}-S I f_{2 n}\right)+\rho\left(S I f_{2 n}-S h\right)\right],
\end{aligned}
$$

which on taking the limit as $n \rightarrow \infty$ implies that $\rho(I h-S h)=0$, that is, $I h-S h=0$ and $I h=S h$. Similarly,

$$
\rho(J h-T h) \leq \omega(3)\left[\rho\left(J h-J T f_{2 n}\right)+\rho\left(J T f_{2 n}-T J f_{2 n}\right)+\rho\left(T J f_{2 n}-T h\right)\right],
$$

which on taking the limit as $n \rightarrow \infty$ implies that $\rho(J h-T h)=0$, that is, $J h-T h=0$ and $J h=T h$. Next we show that $J h=I h$. Assume to the contrary $J h \neq I h$, that is, $\rho(I h-J h)>0$. By the given assumption, we have

$$
\rho(I h-J h)=\rho(S h-T h) \leq \alpha \rho(I h-J h) ;
$$

a contradiction. Hence $J h=I h$, therefore, $S h=T h=I h=J h$. Hence $h$ is a coincidence point of $S, I, T$, and $J$.

Corollary 3.6 Let $(X, \preceq, \rho)$ be a complete ordered modular function space and $S$ and $T$, continuous self-maps on $X_{\rho},(S, T)$ and $(T, S)$ are $\rho$-partially weakly increasing with respect 
to identity mapping on $X$, and

$$
\rho(S f-T g) \leq \alpha \rho(f-g)
$$

holds for every $f, g \in X_{\rho}$ for which $f$ and $g$ are comparable. Then the pair $(S, T)$ has a common fixed point.

Corollary 3.7 Let $(X, \preceq, \rho)$ be a complete ordered modular function space and $S$ and $J$ continuous self-maps on $X_{\rho}$. Suppose that $(S, J)$ is $\rho$-compatible, $S$ is $\rho$-partially weakly increasing with respect to $J$, and

$$
\rho(S f-S g) \leq \alpha \rho(J f-J g)
$$

holds for every $f, g \in X_{\rho}$ for which $J f$ and $J g$ are comparable. Then the pair $(S, J)$ has a coincidence point $h \in X$.

Example 3.8 Assume that $X_{\rho}=\ell^{1}$, where $\rho(x)=\|x\|$ for $x \in \ell^{1}$. For $x, y \in \ell^{1}$, define $x \preceq y$ if and only if $x \geq y$.

Let $S, J: \ell^{1} \rightarrow \ell^{1}$ be defined as

$$
\begin{aligned}
& S(x)=\left(\frac{1}{6} x_{1}, \frac{1}{6} x_{2}, 0,0,0, \ldots\right), \\
& J(x)=\left(\frac{1}{3} x_{1}, \frac{1}{3} x_{2}, 0,0,0, \ldots\right) .
\end{aligned}
$$

Note that

$$
\begin{aligned}
\rho(S x-S y) & =\|S x-T y\|=\left\|\left(\frac{1}{6} x_{1}-\frac{1}{6} y_{1}, \frac{1}{6} x_{2}-\frac{1}{6} y_{2}, 0,0, \ldots\right)\right\| \\
& =\frac{1}{6}\left[\left|x_{1}-y_{1}\right|+\left|x_{2}-y_{2}\right|\right] \leq \frac{2}{9}\left[\left|x_{1}-y_{1}\right|+\left|x_{2}-y_{2}\right|\right] \\
& =\frac{2}{3}\left[\frac{1}{3}\left|x_{1}-y_{1}\right|+\frac{1}{3}\left|x_{2}-y_{2}\right|\right]=\frac{2}{3} \rho(J x-J y) .
\end{aligned}
$$

That is,

$$
\rho(S x-T y) \leq \frac{2}{3} \rho(J x-J y)
$$

Note that $\rho$-compatibility of $(S, J)$ follows immediately. Also $S$ is $\rho$-weakly partially increasing with respect to $J$. Indeed, $S\left(X_{\rho}\right) \subseteq J\left(X_{\rho}\right)$ and $S f \preceq S g$ for all $g \in J^{-1}(S f)$. Therefore all conditions of Corollary 3.7 are satisfied. However, the pair $(S, J)$ has $(0,0,0, \ldots, 0, \ldots)$ as a coincidence and a common fixed point.

\section{Periodic point results}

If $S$ is a map which has a fixed point $f$, then $f$ is also a fixed point of $S^{n}$ for every natural number $n$. However, the converse is false. If a map satisfies $F(S)=F\left(S^{n}\right)$ for each $n \in N$, where $F(S)$ denotes a set of all fixed point of $S$, then it is said to have property $P$ [23]. We 
shall say that $S$ and $T$ have property $Q$ if $F(S) \cap F(T)=F\left(S^{n}\right) \cap F\left(T^{n}\right)$. The set $O(T, \infty)=$ $\left\{f, T f, T^{2} f, \ldots\right\}$ is called the orbit of $T$. The set $O(T, S, \infty)=\left\{f, T f, S f, T^{2} f, S^{2} f, \ldots\right\}$ is called the orbit of $T$ and $S$.

As an application of our results in Section 2, we provide the following periodic point theorems.

Theorem 4.1 Let $S$ be a non-decreasing self-map of a complete ordered modular function space $(X, \preceq, \rho)$, satisfying

$$
\rho\left(S f-S^{2} f\right) \leq \alpha \rho(f-S f)
$$

for all $f \in X$, or (ii) with strict inequality, $\alpha=1$ and for all $f \in X, f \neq S f$. If, $F(S) \neq \phi$, then $S$ has property $P$ provided that $f \preceq S f$ for any $f \in F\left(S^{n}\right)$.

Proof We shall always assume that $n>1$, since the statement for $n=1$ is trivial. Let $f \in F\left(S^{n}\right)$. Then $f \preceq S f$, so a non-decreasing characteristic of the mapping $S$ implies that $O(f, \infty)$ is a well-ordered subset of $X$. Suppose that $S$ satisfies (i). Then

$$
\begin{aligned}
\rho(f-S f) & =\rho\left(S\left(S^{n-1} f\right)-S^{2}\left(S^{n-1} f\right)\right) \\
& =\rho\left(S^{2}\left(S^{n-1} f\right)-S f\right) \\
& \leq \alpha \rho\left(S^{n-1} f-S^{n} f\right) \\
& \leq \alpha^{2} \rho\left(S^{n-2} f-S^{n-1} f\right) \\
& \leq \cdots \leq \alpha^{n} \rho(f-S f) .
\end{aligned}
$$

Now the right-hand side of the above inequality approaches zero as $n \rightarrow \infty$. Hence $\rho(f-$ $S f)=0$, and $f=S f$. Suppose that $S$ satisfies (ii). If $S f=f$, then there is nothing to prove. Suppose, if possible, that $S f \neq f$. Then a repetition of the argument for case (i) leads to

$$
\rho(f-S f)<\rho(f-S f)
$$

a contradiction. Therefore, in all cases, $f=S f$.

Theorem 4.2 Let $(X, \preceq, \rho)$ be a complete ordered modular function space. Let the mappings $S$ and $T$ be as in Corollary 3.6. Then $S$ and $T$ have property $Q$ provided that $O(T, S, \infty)$ for every $f \in F\left(S^{n}\right) \cap F\left(T^{n}\right)$.

Proof From Corollary 3.6, $S$ and $T$ have a common fixed point in $X$. Let $f \in F\left(S^{n}\right) \cap F\left(T^{n}\right)$. Now,

$$
\begin{aligned}
\rho(f-S f) & =\rho\left(T\left(T^{n-1} f\right)-S\left(S^{n} f\right)\right) \\
& \leq \alpha \rho\left(T^{n-1} f-S^{n} f\right)=\alpha \rho\left(T^{n-1} f-f\right),
\end{aligned}
$$

and we have

$$
\rho(f-S f) \leq \alpha \rho\left(T^{n-1} f-f\right) \leq \cdots \leq \alpha^{n} \rho(f-S f) .
$$


Now the right-hand side of the above inequality approaches zero as $n \rightarrow \infty$. Hence $\rho(f-$ $S f)=0$, and $f=S f$. Now,

$$
\rho(f-T f)=\rho(S f-T f) \leq \alpha \rho(f-f)
$$

give $\rho(f-T f)=0$, and $f=T f$.

Remark Recently, Paknazar et al. [24] gave the existence of the solutions of the integral equations in modular function spaces. Hajji and Hanebaly [25] also applied their fixed point result to obtain the solution of perturbed integral equations in modular function spaces (see also [26]). Our results can also be employed to solve such integral equations in the framework of complete ordered modular function spaces.

\section{Competing interests}

The authors declare that they have no competing interests.

\section{Authors' contributions}

All authors contributed equally and significantly in writing this paper. All authors read and approved the final manuscript.

\section{Author details}

${ }^{1}$ Department of Mathematics and Applied Mathematics, University of Pretoria, Lynnwood road, Pretoria, 0002, South Africa. ${ }^{2}$ National College of Business Administration and Economics, 40-E1, Gulberg 03, Lahore, Pakistan. ${ }^{3}$ Department of Mathematics, Faculty of Science, King Mongkut's University of Technology Thonburi (KMUTT), Bang Mod, Thrung Khru, Bangkok, 10140, Thailand.

\section{Acknowledgements}

The authors were supported by the Higher Education Research Promotion and National Research University Project of Thailand, Office of the Higher Education Commission (NRU-CSEC No. NRU56000508). The authors are also thankful to the reviewers for their suggestions and remarks, which improved the presentation of this paper.

\section{Received: 16 June 2013 Accepted: 31 January 2014 Published: 17 Feb 2014}

\section{References}

1. Nakano, H: Modulared Semi-Ordered Spaces. Maruzen, Tokyo (1950)

2. Musielak, J, Orlicz, W: On modular spaces. Stud. Math. 18, 591-597 (1959)

3. Ran, ACM, Reurings, MCB: A fixed point theorem in partially ordered sets and some applications to matrix equations. Proc. Am. Math. Soc. 132, 1435-1443 (2004)

4. Nieto, JJ, Rodriguez-Lopez, R: Contractive mapping theorems in partially ordered sets and applications to ordinary differential equations. Order 22, 223-239 (2005)

5. Nieto, JJ, Rodriguez-Lopez, R: Existence and uniqueness of fixed point in partially ordered sets and applications to ordinary differential equations. Acta Math. Sin. 23(12), 2205-2212 (2007)

6. Khamsi, MA, Kozolowski, WK, Reich, S: Fixed point theory in modular function spaces. Nonlinear Anal. 14, 935-953 (1990)

7. Benavides, TD, Khamsi, MA, Samadi, S: Asymptotically regular mappings in modular function spaces. Sci. Math. Jpn. 53, 295-304 (2001)

8. Khamsi, MA: A convexity property in modular function spaces. Math. Jpn. 44, 269-279 (1996)

9. Kuaket, K, Kumam, P: Fixed points of asymptotic pointwise contractions in modular spaces. Appl. Math. Lett. 24, 1795-1798 (2011)

10. Mongkolkeha, C, Kumam, P: Fixed point and common fixed point theorems for generalized weak contraction mappings of integral type in modular spaces. Int. J. Math. Math. Sci. 2011, Article ID 705943 (2011)

11. Mongkolkeha, C, Kumam, P: Common fixed points for generalized weak contraction mappings in modular spaces. Sci. Math. Jpn. e-2012, 117-127 (2012)

12. Mongkolkeha, C, Kumam, P: Some fixed point results for generalized weak contraction mappings in modular spaces. Int. J. Anal. 2013, Article ID 247378 (2013). doi:10.1155/2013/247378

13. Kumam, P: Fixed point theorem for non-expansive mappings in modular spaces. Arch. Math. 40, 345-353 (2004)

14. Kutbi, MA, Latif, A: Fixed points of multivalued mappings in modular function spaces. Fixed Point Theory Appl. 2009, Article ID 786357 (2009)

15. Abbas, M, Khamsi, MA, Khan, AR: Common fixed point and invariant approximation in hyperbolic ordered metric spaces. Fixed Point Theory Appl. 2011, 25 (2011)

16. Abbas, M, Khan, AR, Nemeth, SZ: Complementarity problems via common fixed points in vector lattices. Fixed Point Theory Appl. 2012, 60 (2012)

17. Abbas, M, Nazir, T, Radenovic, S: Common fixed points of four maps in partially ordered metric spaces. Appl. Math Lett. 24, 1520-1526 (2011) 
18. Altun, I, Damjanovic, B, Djoric, D: Fixed point and common fixed point theorems on ordered cone metric spaces. Appl. Math. Lett. 23, 310-316 (2010)

19. Esmaily, J, Vaezpour, SM, Rhoades, BE: Coincidence point theorem for generalized weakly contractions in ordered metric spaces. Appl. Math. Comput. 219, 1536-1548 (2012)

20. Harandi, AA, Emami, H: A fixed point theorem for contraction type maps in partially ordered metric spaces and application to ordinary differential equations. Nonlinear Anal. 72, 2238-2242 (2010)

21. Kozlowski, WM: Modular Function Spaces. Dekker, New York (1988)

22. Khamsi, MA: Fixed point theory in modular function spaces. In: Recent Advances on Metric Fixed Point Theory, pp. 31-58. Universidad de Sevilla, Sevilla (1996)

23. Jeong, GS, Rhoades, BE: Maps for which $F(T)=F\left(T^{n}\right)$. Fixed Point Theory Appl. 6, 87-131 (2005)

24. Paknazar, M, Eshaghi, M, Cho, YJ, Vaezpour, SM: A Pata-type fixed point theorem in modular spaces with application. Fixed Point Theory Appl. 2013, 239 (2013)

25. Hajji, A, Hanebaly, E: Perturbed integral equations in modular function spaces. Electron. J. Qual. Theory Differ. Equ. 2003, 20 (2003)

26. Taleb, AA, Hanebaly, E: A fixed point theorem and its application to integral equations in modular function spaces. Proc. Am. Math. Soc. 127, 2335-2342 (1999)

10.1186/1029-242X-2014-78

Cite this article as: Abbas et al.: Common fixed points in partially ordered modular function spaces. Journal of Inequalities and Applications 2014, 2014:78

\section{Submit your manuscript to a SpringerOpen ${ }^{\ominus}$ journal and benefit from:}

- Convenient online submission

- Rigorous peer review

- Immediate publication on acceptance

- Open access: articles freely available online

- High visibility within the field

- Retaining the copyright to your article 